\title{
Recent Achievements on Passive and Beam Steering Transmitarrays at Millimeter Waves
}

\author{
Orestis Koutsos ${ }^{1,2, \#, ~ R e d a ~ M a d i ~}{ }^{1,2, \#, ~ F r a n c e s c o ~ F o g l i a ~ M a n z i l l o ~}{ }^{1}$, Maciek Smierzchalski ${ }^{1}$, Antonio Clemente ${ }^{1}$, and \\ Ronan Sauleau ${ }^{2}$ \\ ${ }^{1}$ CEA-Leti, Univ. Grenoble-Alpes, F-38000 Grenoble, France \\ ${ }^{2}$ Univ Rennes, CNRS, IETR - UMR 6164, F-35000 Rennes, France \\ email: antonio.clemente@cea.fr, ronan.sauleau@univ-rennes1.fr \\ \# both authors contributed equally to this publication
}

\begin{abstract}
This paper reports an overview of recent advancements on transmitarray antennas at CEA-Leti in collaboration with IETR. Two fixed-beam and an electronicallysteerable prototypes are presented. The first fixed-beam transmitarray radiates orthogonal linear polarizations at $\mathrm{K} / \mathrm{Ka}-$ band. The second achieves wideband high-gain performance at $300 \mathrm{GHz}$ using low-cost vialess technology. The reconfigurable transmitarray leverages on an efficient and low-power solution to attain beam steering and switchable circular polarization at Ka-band. Excellent scanning capabilities in a field of view of $\pm \mathbf{6 0}^{\circ}$, with low axial ratio are experimentally demonstrated.
\end{abstract}

Keywords - Transmitarrays, beam-forming, Ka-band, 300 GHz, polarization agility, electronically scanned antenna.

\section{INTRODUCTION}

Fixed-beam and electronically steerable transmitarrays (TAs) are attractive antenna architectures up to $300 \mathrm{GHz}$ [1][3] for a huge number of applications requiring high gain. Thanks to their spatial feeding mechanism, these systems do not require lossy feed networks. Furthermore, they do not suffer from feed blockage. The fabrication of a TA is compatible with planar processes, such as Printed Circuit Board (PCB) technology, providing a cost-effective solution. $\mathrm{P}-\mathrm{i}-\mathrm{n}$ diodes [4]-[5] or other switches can be integrated on the antenna aperture to enabling electronic beam forming.

Unit-cells (UCs) based on Antenna-Filter-Antenna (AFA) [2],[6] or Frequency-Selective-Surface (FSS) [3],[7] are typically used to design TAs. AFA architectures with metallized vias have been employed up to $140 \mathrm{GHz}$ [6]. They are attractive when advanced functionalities such as dualband/dual-polarization and/or beam-forming are required. Instead, FSS UCs are an excellent option for wideband operation (> 20\%). When TA is design at extremely high mmWave frequency (> $200 \mathrm{GHz}$ ), FSS-based UCs can also mitigate the impact of the fabrication constraints on the antenna behavior [3],[7]. We present here three recent TAs operating at Ka-band and at $300 \mathrm{GHz}$. They achieve enhanced functionalities and performance over the state of the art.

\section{TRANSMITARRAYS AT Ka-BAND}

\section{A. Dual-Band/Dual-Linearly-Polarized Transmitarray}

A shared-aperture dual-band/dual linearly polarized AFA UC design has been proposed in [6]. If compared to the interleaving strategy, which is typically used to implement multi-band TA, the advantage of the proposed design methodology is the possibility to minimize the period of the UC. Consequently, shared-aperture UCs can be selected to achieve enhanced aperture efficiency and improved field of view, in the case of a reconfigurable TA.

The proposed UC, operating at $\mathrm{K} / \mathrm{Ka}-\mathrm{bands}$, is represented in Fig. 1(a). Its overall size equals to $0.48 \lambda_{u l} \times 0.48 \lambda_{u l}$, where $\lambda_{u l}$ stands for the free space wavelength at $29 \mathrm{GHz}$. Note that, this period corresponds to $0.32 \lambda_{d l} \times 0.32 \lambda_{d l}$, with $\lambda_{d l}$ defining the free space wavelength at $19 \mathrm{GHz}$. Four U-shaped slot loaded patch antennas are stacked to realize the required dualband/dual linear polarization. An additional inner layer is used to realize a ground plane. The UC achieves a 1-bit phase quantization by rotating the transmitting layers of an angle of $180^{\circ}$ around the central metallized via connecting the four patch antennas. A $40 \times 40$ TA illuminated using two horns located at the same focal plane $(F / D=0.57)$ has been fabricated to validate the proposed design, see Fig. 1(b). The focal distance and the TA K/Ka-band phase distributions have been optimized to maximize the aperture efficiency. The measured frequency response is plotted in Fig. 1(c). The measured peak gain for the lower and upper frequency are 27.10 and $29.92 \mathrm{dBi}$, respectively.

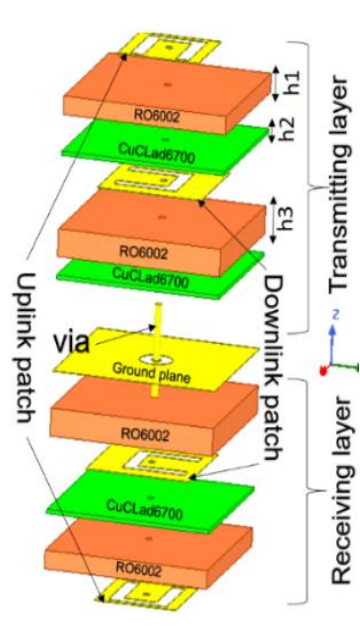

(a)

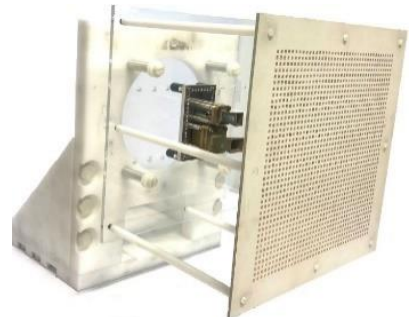

(b)

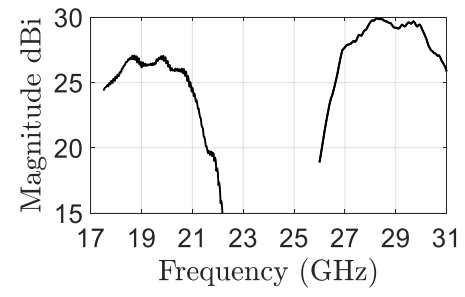

(c)
Fig. 1. TA at K/Ka-band: (a) unit-cell view, (b) prototype, and (c) measured gain frequency response. 


\section{B. Electronically Steerable Transmitarray with Switchable Circular Polarization}

Electronically reconfigurable TAs using simple switches such as $\mathrm{p}$-i-n diodes offer an energy-efficient solution for beam-forming at Ka-band, especially when high gain (> 25 $\mathrm{dBi}$ ) and wideband features are required. In general, these architectures cannot provide both electronically beamscanning and polarization agility without increasing the antenna complexity (phase resolution, layers, number of switches and bias lines).

A 24×24-element TA, shown in Fig. 2(a), overcoming these challenges, while maintaining a good aperture efficiency, has been recently demonstrated by CEA-Leti [5]. It combines an equal number of vertically and horizontally polarized 2-bit AFA UCs with four $\mathrm{p}-\mathrm{i}-\mathrm{n}$ diodes [4]. A random UC distribution has been optimized to generate a switchable circular polarization $(\mathrm{CP})$ with an axial ratio at broadside $<0.5$ $\mathrm{dB}$ at $29.25 \mathrm{GHz}$. The TA scans in all the azimuthal planes in an angular range of $\pm 60^{\circ}$ for both RHCP and LHCP (see Fig. (b)) with a maximum gain of $24.0 \mathrm{dBi}$. At broadside, the measured axial ratio is $<1 \mathrm{~dB}$ between 28.9 and $31.2 \mathrm{GHz}$.

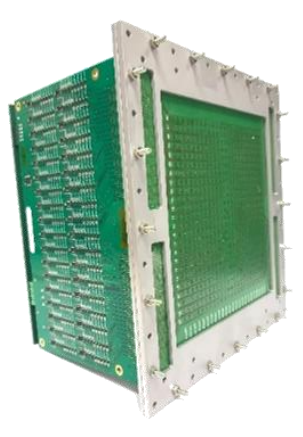

(a)

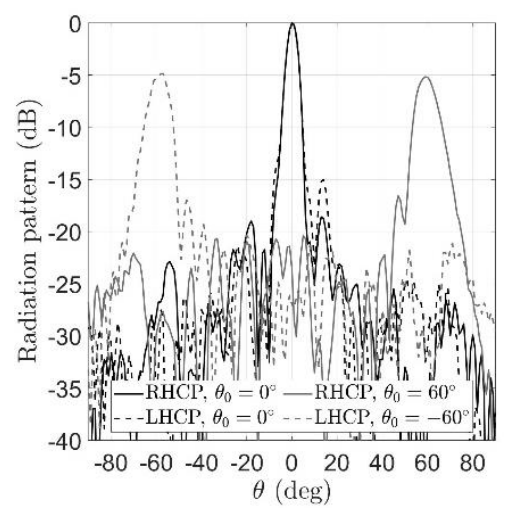

(b)
Fig. 2. (a) Prototype of the electronically steerable TA at Kaband. (b) A selection of measured beams at $29.25 \mathrm{GHz}$.

\section{TRANSMITARRAYS AT SUB-THz}

A 3-bit TA operating at $300 \mathrm{GHz}$ has been optimized and prototyped by using standard PCB technology, see Fig. 3(a). A three-layer FSS-based UC (Fig. 3(b)) with no metallized vias is employed to mitigate the impact of fabrication constraints on the TA performance. Based on the synthesis procedure proposed in [7], a wideband transmission with 3-bit phase quantization is achieved by opportunely selecting the element printed on the inner layer.

A $40 \times 40$ TA illuminated by a $10-\mathrm{dBi}$ horn has been optimized $(F / D=1)$. The simulated and measured results are in good agreement, see Fig. 3(c). A peak gain and a relative 3$\mathrm{dB}$ bandwidth of $32.2 \mathrm{dBi}$ and $25.1 \%$ have been, respectively, demonstrated. The prototype outperforms state-of-the-art TA antennas operating at frequencies higher than $200 \mathrm{GHz}$.

\section{CONCLUSION}

Three state-of-the-art TAs, operating at millimeter wave frequencies, have been described in this paper. All the TAs have been designed in standard PCB technology. A dualband/dual polarized TA operating at $\mathrm{K} / \mathrm{Ka}$-band and an electronically steerable TA with switchable CP have been proposed as possible candidates for future low-cost and energy-efficient SATCOM terminal antennas. Both TAs are based on advanced AFA UC architectures. The third TA comprises three-layer vialess FSS-based UCs. It operates at $300 \mathrm{GHz}$ for future high-data-rate communications.

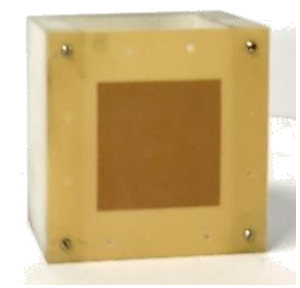

(a)

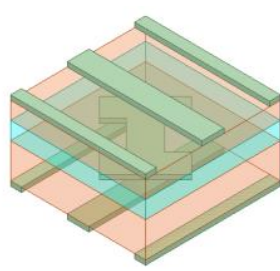

(b)

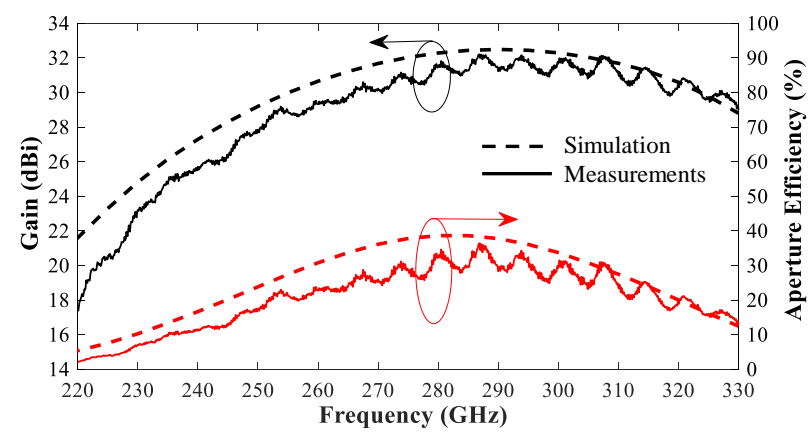

(c)

Fig. 3. (a) TA prototype at $300 \mathrm{GHz}$, (b) unit-cell view and (c) simulated and measured gain and aperture efficiency.

\section{ACKNOWLEDGMENT}

This work was partly supported by the National Research Agency (ANR) through the project "Next5G" and "ArtiKa" under Grants ANR 18-CEA25-0009-01 and ANR-20-ASTR0014-01.

\section{REFERENCES}

[1] J. R. Reis, M. Vala and R. F. S. Caldeirinha, "Review Paper on transmitarray antennas," in IEEE Access, vol. 7, pp. 94171-94188, 2019.

[2] F. Foglia Manzillo, A. Clemente, and J. L. Gonzalez-Jimenez, "Highgain D-band transmitarray in standard PCB technology for beyond5G communications," IEEE Trans. Antennas Propag., vol. 68, no. 1, pp. 587-592, Jan. 2020.

[3] K. Medrar, et al, "H-band substrate-integrated discrete lens antenna for high data rate communication systems," IEEE Trans. Antennas Propag., in press.

[4] F. Diaby, A. Clemente, R. Sauleau, K. Pham, and L. Dussopt, "2-bit reconfigurable unit-cell and electronically steerable transmitarray at Ka-band," IEEE Trans. Antennas Propag., vol. 68, no. 6, pp. 50035008, Jun. 2020.

[5] F. Foglia Manzillo, M. Smierzchalski, J. Reverdy, and A. Clemente, "A Ka-band beam-steering transmitarray achieving dual-circular polarization," in Proc. 15 $15^{\text {th }}$ Eu. Conf. Antennas Propag. (EuCAP 2021), Dusseldorf, Germany, Mar. 2021.

[6] R. Madi, A. Clemente, and R. Sauleau, "Dual-band dual-linearly polarized transmitarray at Ka-band," in Proc. 50th Eu. Microwave Conf. (EuMC 2020), Utrecht, Netherlands, Jan. 2021.

[7] O. Koutsos, F. F. Manzillo, A. Clemente and R. Sauleau, "Analysis and efficient design of sub-THz transmitarrays with three anisotropic layers," in Proc. 15 $15^{\text {th }}$ Eu. Conf. Antennas Propag. (EuCAP), Dusseldorf, Germany, Mar. 2021. 